\title{
The South Pole Telescope: Latest Results and Future Prospects
}

\author{
Bradford Benson $^{1}$ \& the SPT Collaboration (pole.uchicago.edu) \\ ${ }^{1}$ Kavli Institute for Cosmological Physics, University of Chicago \\ 5640 South Ellis Avenue, Chicago, IL 60637 \\ email: bbenson@kicp. uchicago.edu
}

\begin{abstract}
The South Pole Telescope is a 10 meter telescope optimized for sensitive, highresolution measurements of the cosmic microwave background (CMB) anisotropy and millimeterwavelength sky. In November 2011, the SPT completed the $2500 \mathrm{deg}^{2}$ SPT-SZ survey. The survey has led to several major cosmological results, derived from measurements of the fine angular scale primary and secondary CMB anisotropies, the discovery of galaxy clusters via the Sunyaev-Zel'dovich (SZ) effect and the resulting mass-limited cluster catalog, and the discovery of a population of distant, dusty star forming galaxies (DSFGs). In January 2012, the SPT was equipped with a new polarization sensitive camera, SPTpol, which will enable detection of the contribution to the CMB polarization power spectrum from lensing by large scale structure (the so-called "lensing $B$-modes") and, on larger angular scales, a detection or improved upper limit on the primordial inflationary signal ("gravitational-wave $B$-modes"), thereby constraining the energy scale of Inflation. Development is underway for SPT-3G, the third-generation camera for SPT. The SPT-3G survey will cross the threshold from statistical detection of $B$-mode CMB lensing to imaging the fluctuations at high signal-to-noise; enabling the separation of lensing and inflationary $B$-modes and improving the constraint on the sum of the neutrino masses $\Sigma m_{\nu}$ to a level relevant for exploring the neutrino mass hierarchy.
\end{abstract}

Keywords. cosmology, cosmic microwave background, clusters of galaxies, polarization

\section{Introduction}

The South Pole Telescope (SPT, Fig. 1) is a 10 meter telescope optimized for lownoise, high-resolution imaging surveys of the sky at millimeter ( $\mathrm{mm}$ ) and submillimeter (submm) wavelengths. In particular, all aspects of the SPT - the site, the telescope, the $\mathrm{RF}$ shielding and the cryogenic receivers - have been optimized for making ultra-sensitive measurements of the cosmic microwave background (CMB) anisotropy from degree to arcminute angular scales over thousands of square degrees of the sky (Carlstrom et al. 2011). The telescope is located at the NSF Amundsen-Scott South Pole station, the best developed site on Earth for mm-wave observations, with 30 times less atmospheric fluctuation power than found at the ALMA site in the Atacama desert (Bussman et al. 2005, Radford 2011). The telescope is an off-axis, classical Gregorian design which gives a wide diffraction-limited field of view, low scattering and high efficiency with no blockage of the primary aperture. The current telescope optics produce a $1^{\prime}$ FWHM beam at $150 \mathrm{GHz}$ with a conservative illumination of the inner 8 meters of the telescope and a $\sim 1 \operatorname{deg}^{2}$ diffraction-limited field of view (Padin et al. 2008). The SPT is designed to

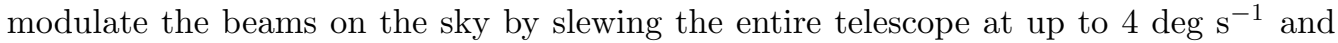
eliminating the need for a chopping mirror. The SPT observing program consists of three planned, underway, or proposed surveys: 1) SPT-SZ (2007-2011), 2) SPTpol (2012-2015) and 3) SPT-3G (2016-2019). 

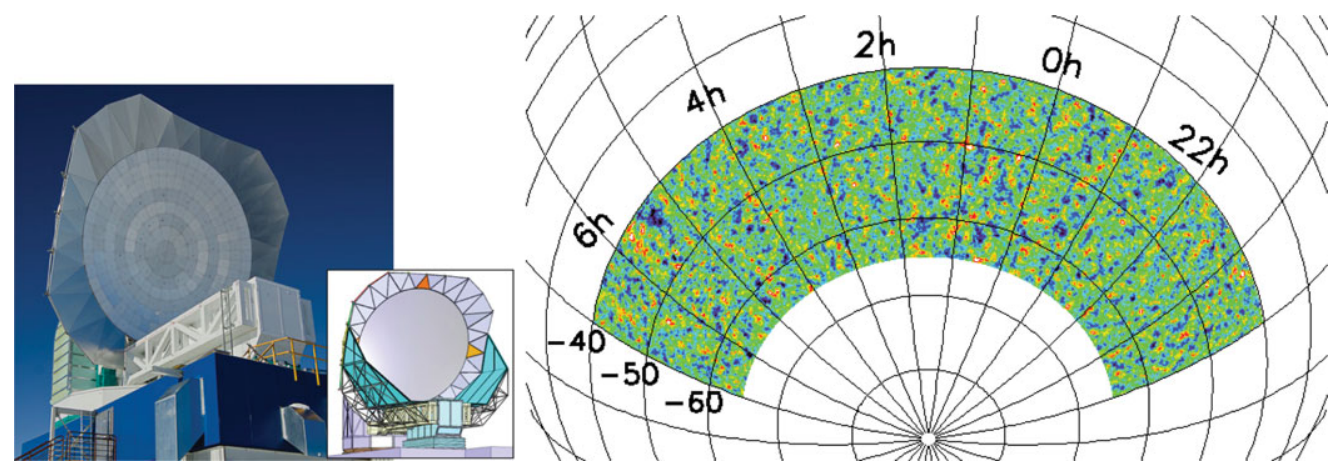

Figure 1. (Left) Photograph of the SPT with a new RF shield extending past the 10 meter primary. The inset shows the mechanical design of the second component of new shielding to be installed in November 2012. (Right) The $2500 \mathrm{deg}^{2}$ SPT-SZ survey map at $95 \mathrm{GHz}$.

\section{SPT-SZ}

The SPT-SZ survey was completed in November 2011 and covers $2500 \mathrm{deg}^{2}$ of the sky (see Fig. 1) at 95, 150 and $220 \mathrm{GHz}$ with unprecedented depth and angular resolution. The SPT-SZ observations have led to significant results and new discoveries in three main areas: using the SZ effect to discover new galaxy clusters (particularly at high redshift), measurements of fine-scale CMB anisotropy, and the systematic discovery of strongly lensed high-redshift star forming galaxies.

The SPT-SZ cluster survey produced the first discovery of galaxy clusters via the SZ effect (Staniszewski et al. 2009), the first cosmological constraints from an SZ cluster survey (Vanderlinde et al. 2010), and constraints on primordial non-Gaussianity from the most massive clusters in the survey (Williamson et al. 2011). Benson et al. (2012) was the first result to use an SZ cluster survey to demonstrate significant improvements on the dark energy equation of state, $w$, and the sum of the neutrino masses, $\Sigma m_{\nu}$, measuring $w=-0.973 \pm 0.063$ and $\Sigma m_{\nu}<0.28 \mathrm{eV}$ at $95 \%$ confidence, a factor of 1.25 and 1.4 improvement, respectively, over the constraints without SPT cluster data. Reichardt et al. (2012a) released a catalog of 158 SZ-selected optically confirmed clusters (see Fig. 2) from the first $720 \mathrm{deg}^{2}$ of the SPT-SZ survey, more than doubling the number of comparably massive clusters discovered at redshift $z>0.5$. The unique sample of massive, high-redshift clusters discovered in the SPT-SZ survey has motivated numerous follow-up observations spanning wavebands from radio to X-rays, with large programs on the Herschel, Spitzer, Hubble, XMM-Newton and Chandra space telescopes.

The high sensitivity and angular resolution of the SPT-SZ survey are also ideal for precision measurements of fine-scale CMB anisotropy, and have led to many significant results. The SPT-SZ survey made the first detection of secondary CMB anisotropy due to the background of lower mass SZ clusters (Lueker et al. 2010), the first CMB-based constraint on the evolution of the ionized fraction during the epoch of reionization (Reichardt et al. 2012b, Zahn et al. 2012), the most-significant detection of non-Gaussian power in the CMB from gravitational lensing (Van Engelen et al. 2012), and the first detection of galaxy bias from CMB-lensing (Bleem et al. 2012). The SPT-SZ survey has also led to the best current measurement of the primordial fine-scale CMB anisotropy power spectrum (Fig. 2, Keisler et al. 2011). These data are sensitive to the expansion rate during recombination and thus to the number of relativistic particle species present at that epoch. This has allowed SPT to place the tightest yet constraints on the number of light particle species beyond the standard three neutrinos (e.g., sterile neutrinos). 

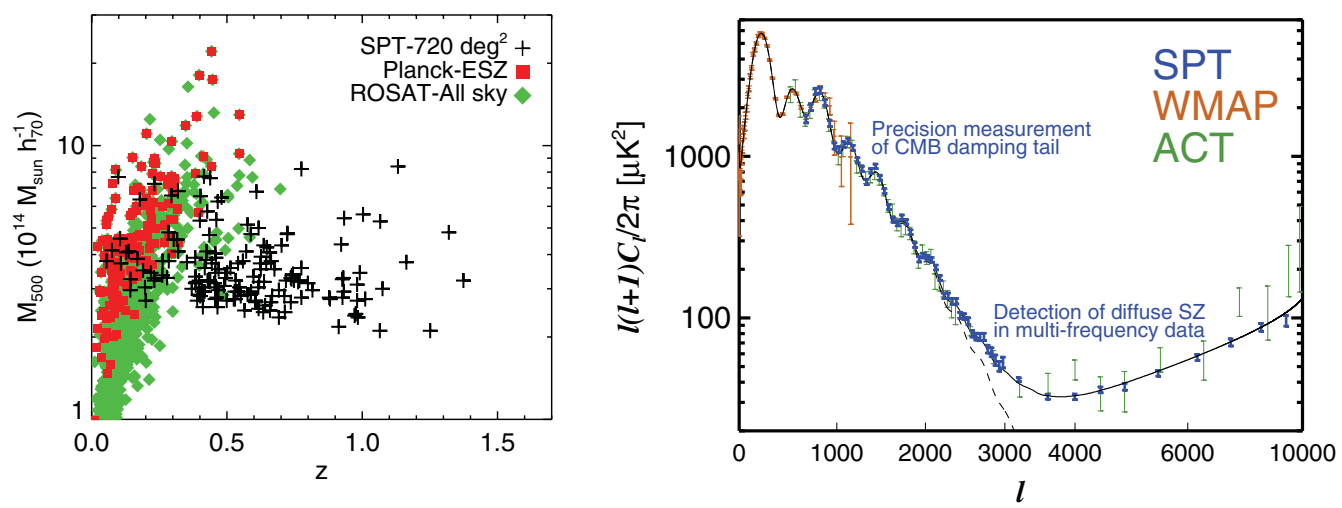

Figure 2. (Left) Mass vs. redshift for three cluster samples: (1) SZ-selected clusters from 720 $\mathrm{deg}^{2}$ of the SPT-SZ survey, (2) SZ-selected clusters from Planck, and (3) X-ray selected clusters from the ROSAT all-sky survey. High-resolution SZ surveys, such as SPT-SZ, have a nearly redshift-independent selection. (Right) SPT measurement of the CMB power spectrum using $790 \mathrm{deg}^{2}$, or $1 / 3$ of the full SPT-SZ survey, along with data from WMAP and ACT. A best-fit $\Lambda \mathrm{CDM}$ theory spectrum is shown with dashed (CMB) and solid (CMB+foregrounds) lines.

\section{SPTpol}

On January 27, 2012, first light on SPT was achieved for SPTpol, a new polarization sensitive camera with significantly higher mapping speed and, crucially, polarization sensitivity. The 768-pixel SPTpol focal plane contains detectors at two observing frequencies, 90 and $150 \mathrm{GHz}$, composed of two different detector architectures fabricated at Argonne National Lab and NIST, respectively, in collaboration with the SPT team. At $150 \mathrm{GHz}$, SPTpol consists of seven arrays of corrugated feedhorn-coupled TES polarimeters providing 588 dual-polarization pixels, or 1176 total bolometers (Hubmayr et al. 2011). At $95 \mathrm{GHz}$, SPTpol uses 180 individually packaged dual-polarization absorber-coupled polarimeters (a total of 360 bolometers) that are coupled to the telescope through machined contoured feedhorns (Chang et al. 2012). Fig. 3 shows a photo of the focal plane.

Projected constraints on the $B$-mode power spectrum from three-years of SPTpol data are shown in Fig. 3. With Planck priors, this data will lead to a constraint on the tensorto-scalar ratio of $\sigma(r)=0.028$ and a $1 \sigma$ uncertainty on the sum of the neutrino masses $\sigma\left(\Sigma m_{\nu}\right)=0.096 \mathrm{eV}$. This constraint on $\Sigma m_{\nu}$ will be roughly four times better than the KATRIN beta decay experiment, which has a predicted sensitivity of $\sim 0.6 \mathrm{eV}$ (90\% C.L.) for $\Sigma m_{\nu}$ (Gonzalez-Garcia et al. 2010), and compliments other cosmological probes.

\section{SPT-3G}

We are currently developing a third-generation camera for the SPT, SPT-3G, which will exploit two technological advances to achieve a significant leap in sensitivity: 1) an improved wide-field optical design that allows more than twice as many diffractionlimited optical elements, and 2) multi-chroic pixels, sensitive to multiple observing bands in a single detector element. The combination will deliver a factor-of-20 improvement in mapping speed over the already impressive SPTpol camera. In total, the focal plane will consist of 15,234 detectors in three observing bands (90, 150, and $220 \mathrm{GHz}$ ) and will represent a fundamental step forward in CMB polarization measurements.

The SPT-3G camera will enable the advance from statistical detection of B-mode polarization power to high signal-to-noise measurements of the individual modes, i.e., maps. This data set will enable a broad range of cosmological results on dark energy, 

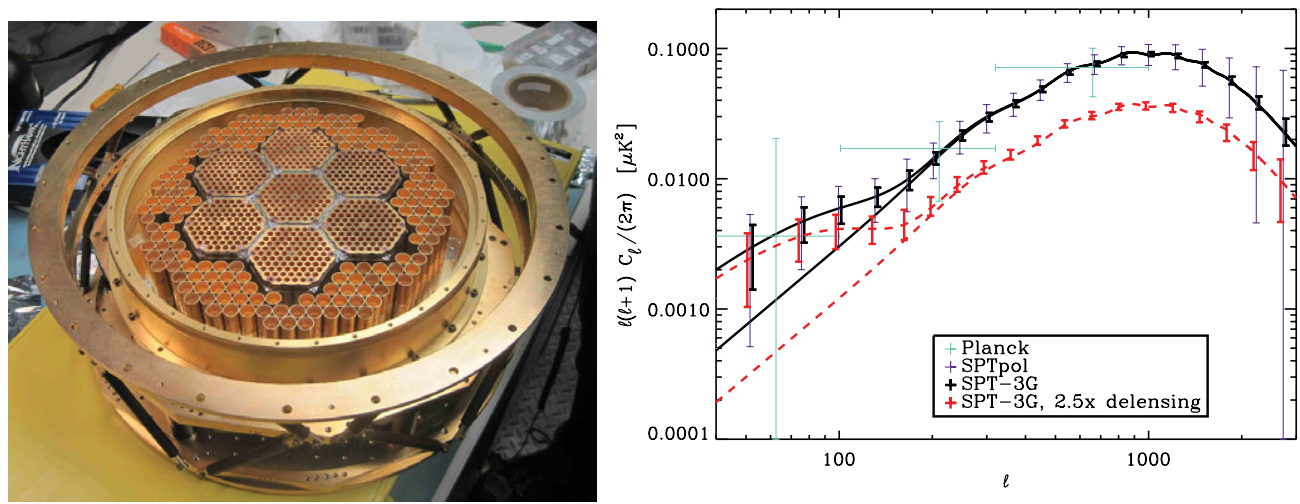

Figure 3. (Left): Photo of the SPTpol focal plane. (Right) Projected B-mode constraints from four years of observing with the SPT-3G camera (black, thick-cross), with overplotted projected constraints from Planck (blue, dashed-cross) and SPTpol (purple, thin-cross). Model curves (solid lines) are for $\Sigma m_{\nu}=0$, with $r=0$ and $r=0.04$. The red-medium-cross and dashed lines show the added sensitivity to primordial gravitational-wave B-modes from de-lensing, which causes a 2.5 reduction in lensed B-mode power.

neutrino mass, General Relativity, the epoch of reionization, and Inflation. For example, the $2500 \mathrm{deg}^{2}$ SPT-3G survey would enable an extremely tight constraint on $r(\sigma(r)<$ 0.01, see Fig. 3), and a constraint on $\Sigma m_{\nu}$ to a level relevant for exploring the mass hierarchy $\left(\sigma\left(\Sigma m_{\nu}\right)<0.06 \mathrm{eV}\right)$. It will also yield a cluster catalog 10 times larger than SPT-SZ, when combined with DES will yield a dark energy figure-of-merit $>100$.

In a broader context, the high-sensitivity and high-resolution mm-wave temperature and polarization data are the first in a series of targeted surveys of the large region of the Southern sky with low Galactic foregrounds. They will inform and complement the upcoming LSST and CCAT surveys, as well as future planned spectroscopic surveys. The SPT-3G survey will uniquely contribute a measurement of the projected mass distribution over the full $2500 \mathrm{deg}^{2}$ region, enabling powerful new probes of large scale structure and adding value to all-sky surveys such as Planck and WISE.

\section{References}

Benson, B. A., et al. 2012, ApJ submitted, arXiv:astro-ph/1112.5435

Bleem, L. E., et al. 2012, ApJ, 753, 9

Bussman, R. S., Holzapfel, W. L., \& Kuo, C. L. 2005, ApJ, 622, 1343

Carlstrom, J. E., et al. 2011, PASP, 123, 568

Chang, C. L., et al. 2012, Journal of Low-Temperature Physics, 183

Gonzalez-Garcia, M., Maltoni, M., \& Salvado, J. 2010, Journal of High Energy Physics, 08, 117

Hubmayr, J., et al. 2012, Journal of Low-Temperature Physics, Jan, 904

Keisler, R., et al. 2011, ApJ, 743, 28

Lueker, M., et al. 2010, ApJ, 719, 1045

Padin, S., et al. 2008, Applied Optics, 47, 24, 4417

Radford, S. 2011, Revista Mexicana de Astronomia, arXiv:astro-ph/1107.5633

Reichardt, C. L., et al. 2012, ApJ submitted, arXiv:astro-ph/1203.5775

Reichardt, C. L., et al. 2012, ApJ, 755, 70

Van Engelen, A., et al. 2012, ApJ, 756, 142

Vanderlinde, K., et al. 2010, ApJ, 722, 1180

Vieira, J. D., et al. 2010, ApJ, 719, 763

Williamson, R., et al. 2011, ApJ, 738, 139

Zahn, O., et al. 2012, ApJ, 756, 65 